Revue d'histoire de l'Amérique française

ZAS REVUE D.HISTOIRE DE L'AMÉRIQUE FRANÇAISE

\title{
Pièces récentes à verser au procès du fédéralisme canadien (1971-1973)
}

\section{Richard Arès}

Volume 28, numéro 2, septembre 1974

URI : https://id.erudit.org/iderudit/303350ar

DOI : https://doi.org/10.7202/303350ar

Aller au sommaire du numéro

Éditeur(s)

Institut d'histoire de l'Amérique française

ISSN

0035-2357 (imprimé)

1492-1383 (numérique)

Découvrir la revue

Citer cette note

Arès, R. (1974). Pièces récentes à verser au procès du fédéralisme canadien (1971-1973). Revue d'histoire de l'Amérique française, 28(2), 245-258.

https://doi.org/10.7202/303350ar d'utilisation que vous pouvez consulter en ligne.

https://apropos.erudit.org/fr/usagers/politique-dutilisation/ 


\section{NOTES CRITIQUES}

\section{PIÈCES RÉCENTES À VERSER AU PROCĖS DU FÉDÉRALISME CANADIEN}

(1971-1973)

\section{I - LA CONTRIBUTION ANGLOPHONE}

Le fédéralisme canadien est toujours en question; en dépit de toutes les conférences fédérales-provinciales et commissions royales, son procès se poursuit sur la place publique. Tant chez les anglophones que chez les francophones, il compte des partisans et des adversaires. Même quand on se dit d'accord avec le principe fédératif lui-même, on s'en prend au régime issu de l'Acte de l'Amérique du Nord britannique de 1867, et plus encore à la manière dont les gouvernements ont vécu ce régime ainsi qu'à l'interprétation qu'en a donnée le Conseil privé de Londres.

Les ouvrages parus au cours des années 1971-1973 illustrent bien ces difficultés que traverse le fédéralisme canadien. Sans doute, les critiques les plus dures viennent-elles surtout du côté francophone, mais, même si elles sont plus rares, elles commencent à se faire jour du côté anglophone.

Parmi une bonne dizaine d'ouvrages publiés en langue anglaise durant ces trois années, j'en choisis ici quelques-uns pour en signaler le contenu et les tendances. Je les divise en quatre catégories: les recueils documentaires, les analyses historiques, les essais politiques et les projets de réforme constitutionnelle.

\section{Les recueils documentaires}

Ces recueils documentaires sont au nombre de deux. Ce sont des ouvrages qui se contentent de republier des textes historiques importants sur des points particuliers, soit sans commentaire, soit avec une présentation de circonstance. Ainsi, M. J. M. Beck a rassemblé dans un volume un nombre impressionnant de textes se rapportant au problème des relations entre le pouvoir

[245]

RHAF, vol. 28, no 2 (septembre 1974) 
central et les pouvoirs provinciaux, de 1867 à nos jours ${ }^{1}$. Une introduction d'à peine trois pages nous présente le contenu, ensuite il n'y a plus que des textes, par exemple, sur la nature du fédéralisme canadien, la distribution des pouvoirs législatifs, l'exercice du pouvoir de taxer et de dépenser, etc. Pour ma part, j'ai été particulièrement heureux de retrouver certains textes familiers contenus dans le chapitre intitulé: "Is the B.N.A. Act A Compact or a Treaty?", en particulier celui de George F. G. Stanley, "Act or Pact ? Another Look at Confederation". tation.

En somme, un excellent ouvrage de références et de consul-

Les mêmes remarques valent, en gros, pour le volume qu'ont publié MM. Howard A. Leeson et Wilfried Vanderelst sur l'évolution du fédéralisme canadien au plan des relations internationales ${ }^{2}$. Chacun des six chapitres s'ouvre par une brève présentation et se continue par un ensemble de textes historiques se rapportant au sujet traité. Un Québécois, par exemple, s'intéressera tout particulièrement au cinquième chapitre, intitulé "The Stormy Sixties: Quebec versus Ottawa", dans lequel se trouvent consignées les principales déclarations, tant d'Ottawa que de Québec, sur les pouvoirs respectifs des gouvernements fédéral et québécois en matière de relations internationales, entre autres l'allocution prononcée, le 13 avril 1965, par le ministre Paul Gérin-Lajoie sur "La personnalité internationale du Québec" devant le corps consulaire de Montréal.

\section{Les analyses historiques}

A côté de ces recueils documentaires, il convient de placer deux ouvrages qui dénotent un louable effort pour présenter objectivement l'évolution du fédéralisme canadien en ces dernières années. Le premier a trait à la diplomatie fédérale-pro-

1 J. M. Beck, ed., The Shaping of Canadian Federalism: Central Authority or Provincial Right? Coll. "Issues in Canadian History" (Toronto, The Copp Clark Publishing Company, 1971), 229 p. - Bien qu'il n'entre dans aucune des quatre catégories mentionnées ici, je m'en voudrais de ne pas au moins signaler l'excellent ouvrage de J. R. Mallory, The Structure of Canadian Government (Toronto, Macmillan of Canada, 1971), $418 \mathrm{p}$. Il s'agit d'une étude sur la nature et le fonctionnement des institutions politiques par lesquelles se gouverne le Canada.

2 Howard A. Leeson et Wilfried Vanderelst, External Affairs and Canadian Federalism: The History of a Dilemma (Toronto-Montreal, Holt, Rinehart and Winston of Canada, 1973), $138 \mathrm{p}$. 
vinciale, plus précisément au rôle et au fonctionnement des conférences fédérales-provinciales, de 1963 à $1971^{3}$. Son auteur, professeur de science politique à Queen's University, Kingston, analyse longuement les événements qui ont entouré ces conférences fédérales-provinciales; il les groupe autour de trois principaux sujets: les plans de pension, les accords fiscaux et la question constitutionnelle. Il étudie en particulier l'attitude des cinq provinces suivantes: Québec, Ontario, Manitoba, Colombie britannique et Nouveau-Brunswick. Il s'efforce de comprendre la conduite du Québec, en particulier lors de la conférence de Victoria, en 1971. Faisant allusion aux propos tenus par certains représentants fédéraux, à savoir qu'en l'occurrence il s'agissait moins de modifier la constitution que de se rencontrer pour prendre conscience des problèmes du fédéralisme canadien et bâtir un consensus national, en sensibilisant, d'une part, les anglophones aux besoins et aux objectifs des francophones et en convainquant, d'autre part, ces derniers à la fois de leur intérêt à rester dans l'union et de la bonne volonté des anglophones à leur égard, l'auteur, pour tout commentaire, se contente de cette phrase mélancolique et pleine de sous-entendus: "If anything, the opposite effect seems to have been produced by $1971 "$ (p. 123).

Sur un sujet neuf, fort compliqué, autant en tout cas que la réalité culturelle, sociale et politique canadienne, comportant en outre des aspects techniques de droit constitutionnel, l'auteur a exercé son talent d'analyse et de clarté; il y a pleinement réussi.

Un autre professeur de science politique, cette fois de l'Université de Toronto, a traité un sujet analogue, à la fois plus vaste parce que portant sur le fédéralisme canadien en général et plus restreint parce que se bornant aux toutes dernières années ${ }^{4}$. L'ouvrage du professeur Smiley comporte sept chapitres, dont deux intéressent spécialement les Canadiens français et le Québec: le chapitre six, "Cultural Duality and Canadian Federalism" et le chapitre sept, "The Compounded Crisis in Canadian Federalism". L'auteur vise à l'objectivité et reconnaît volontiers que la dualité culturelle du peuple canadien est à la fois la principale raison d'être du fédéralisme et la plus puissante menace à sa stabilité.

3 Richard Simeon, Federal-Provincial Diplomacy: The Making of recent Policy in Canada (Toronto, University of Toronto Press, 1972), $324 \mathrm{p}$.

${ }_{4}$ Donald V. Smiley, Canada in Question: Federalism in the Seventies (Toronto, McGraw-Hill Ryerson, 1972), $190 \mathrm{p}$. 
Selon lui, à la crise du fédéralisme canadien il y a trois solutions: ou un Canada bilingue et biculturel, ou un statut spécial au Québec ou la souveraineté-association, qu'il désigne par l'expression "The separate-states and economic union alternative". Il reconnaît que l'avenir du français dépend surtout du Québec et que le prix à payer pour que dure la Confédération est un nouveau partage des pouvoirs législatifs, partage s'appliquant à toutes les provinces. La troisième solution lui paraît inacceptable: la position du gouvernement canadien, surtout sur le plan de la politique économique, serait intolérable, et l'Ontario dominerait encore plus la politique canadienne. Bref, "it would be highly irrational for Canada to contemplate seriously giving an independent Quebec a veto over national economic policies... The dismemberment of Canadian federalism by negotiation and the subsequent creation of a Canada-Quebec economic union is the least available alternative... It is impossible even to conjecture reasonably the substance of relations between Canada and an independent Quebec" (p. 166).

Tel est le point de vue du professeur Smiley, le point de vue d'un scholar anglophone, pour qui le fédéralisme est encore le meilleur régime qui convienne au Canada, même si ce régime aurait grand besoin d'être amélioré.

\section{Les essais politiques}

J'entends par là des ouvrages qui mettent l'accent moins sur les aspects juridiques du régime actuel que sur les problèmes politiques que soulève un Canada composé de régions diverses et de deux communautés linguistiques et culturelles. Inquiets pour l'avenir du Canada, surtout à la suite des événements survenus au Québec en 1970, un groupe d'intellectuels anglophones, provenant pour la plupart de Queen's University, Kingston, ont entrepris, sous la direction du professeur R. M. Burns, de s'interroger sur les faiblesses et lacunes de notre régime politique actuel ainsi que sur les chances de survie de la "nation" canadienne ${ }^{5}$. L'avant-propos dit clairement leur objectif: "The

${ }^{5}$ R. M. Burns, ed., One Country or Two? With an introduction by John J. Deutsch (McGill-Queen's University Press, Montreal and London, 1971), 287 p. - De cet ouvrage se rapprochent deux autres publications: Working Papers on Canadian Politics par John Meisel, sortie des presses des deux mêmes universités en 1972 et reproduisant l'un des textes les plus intéressants parus dans One Country or Two? sur les options actuelles qui s'offrent au Canada, précisément par John Meisel. L'autre rassemble, sous la direction 
central concern of these essays is the effect of Quebec political attitudes upon the various threads of the fabric of Canadian nationality."

Tous nationalistes canadiens, tous fédéralistes, ils s'interrogent honnêtement et cherchent à comprendre les aspirations des Canadiens français et les revendications du Québec. Ils examinent tour à tour le problème général de la survivance et de la désintégration des fédérations, les scénarios pouvant servir à une séparation, le partage de la voie maritime, le sort des Québécois anglophones dans un Québec séparé, les options qui se présentent actuellement au Canada, le sort des Maritimes face à une sécession possible du Québec, etc. En général, ils se montrent favorables aux revendications linguistiques des Canadiens français, mais non aux demandes visant à accorder au Québec soit un statut particulier, soit un nouveau partage des pouvoirs législatifs, lequel, à leurs yeux, nuirait à l'efficacité politique et administrative du gouvernement fédéral.

Le principal de l'Université Queen's, John J. Deutsch, dans l'introduction qu'il a rédigée, reconnaît que l'évolution du fédéralisme a coïncidé en ces dernières années avec des bouleversements sociaux et religieux qui ont affecté surtout le Québec et qu'elle a fait mal aux Canadiens français plus qu'aux autres groupes ethniques. Je signale comme particulièrement intéressants les trois chapitres suivants: "Scenarios for Separation" par Richard Simeon, "English-Speaking Quebeckers in a Separate Quebec" par J. R. Mallory et "Cancel out and Pass on: A View of Canada's Present Options" par John Meisel.

L'ensemble forme un volume qui mérite d'être lu avec attention et sympathie. On y trouve exprimés les sentiments et les convictions d'anglophones qui se disent prêts à dialoguer avec les Canadiens français sur tous les sujets, y compris celui de l'indépendance du Québec. Le fait étant plutôt rare, il me fait plaisir de le signaler ici et de recommander la lecture du volume où il s'est produit.

Je ne peux malheureusement pas en dire autant d'un autre petit volume dû à la plume d'un ancien juge de la Cour de l'Echi-

de Thomas Hockin, des textes de différents auteurs et s'intitule The Canadian Condominium. Domestic Issues and External Policy (Toronto, McClelland and Stewart, 1972), $176 \mathrm{p}$. - J'attire l'attention sur le chapitre 3, qui a pour auteur Ken McRoberts et pour titre, "Quebec: Canada's Special Challenge and Stimulus". 
quier et maintenant président de The Single Canada League ${ }^{6}$. Sa position de base est bien connue, puisqu'il la répète sur toutes les tribunes et jusque devant les tribunaux, y compris la Cour suprême du pays. En peu de mots, la voici, telle qu'on peut la retrouver dans son petit livre: le Canada n'est pas un pays bilingue et tous les efforts pour le rendre tel sont source de division et causent du ressentiment chez des milliers de Canadiens. Il faut, en conséquence, blâmer ceux qui travaillent à modifier le caractère fondamental du Canada pour en faire un pays anglo-français. Les coupables à cet égard sont la Commission royale d'enquête sur le bilinguisme et le biculturalisme, les premiers ministres québécois Lesage et Johnson, les Etats généraux du Canada français, les partisans du statut particulier pour le Québec, les premiers ministres Pearson et Trudeau, ce dernier surtout qui a fait adopter la loi fédérale sur les langues officielles et a imposé le bilinguisme dans les services publics, etc.

Ce qu'il veut, il le dit clairement: "It is a Single Canada, not a dual French-English one" (p. 7). Les Canadiens d'aujourd'hui, écrit-il, ont un choix à faire: ou un Canada anglo-français, tel que le gouvernement tente d'établir, dominé qu'il est par ses membres québécois, ou un seul Canada, tel que les Canadiens de toutes les origines ont entrepris de bâtir, sans aucune préférence pour les membres de quelque groupe que ce soit de la nation canadienne. S'il faut s'inquiéter de l'état présent des rapports entre le Québec et le reste du Canada, il n'en faut pas moins soutenir que les exigences du Québec sont inacceptables et doivent être rejetées.

Dans l'affaire du maire Jones, de Moncton, dont l'ancien juge Thorson était le procureur, la Cour suprême du Canada vient de se prononcer par la négative sur la valeur juridique de ces arguments; quant à leur valeur politique elle me paraît nulle et un pareil combat d'arrière-garde ne peut que susciter, en réaction, la volonté pour le Québec d'être enfin pleinement maître chez lui.

\section{Un essai de réforme constitutionnelle}

Même s'il ne comporte que peu d'aspects historiques et même si la publication qui l'a fait connaître est scrupuleuse-

${ }^{6}$ J. T. Thorson, Wanted: A Single Canada (Toronto, McClelland and Stewart, 1973), $160 \mathrm{p}$. 
ment bilingue, il importe de signaler ici le rapport qu'a produit le Comité parlementaire chargé d'étudier les réformes à faire à la constitution canadienne ${ }^{7}$. Composé de dix sénateurs et de vingt députés, ce Comité a parcouru tout le pays pour entendre les plaintes et les déstrs des citoyens canadiens à ce sujet. Après deux ans de travail, il a remis, en 1972, son rapport, riche de multiples considérations et de 105 diverses recommandations. Il y expose son avis sur le besoin d'une nouvelle constitution, sur l'autodétermination, le partage des pouvoirs, les droits fondamentaux, y compris les droits linguistiques, les institutions fédérales, la politique sociale, la réglementation de l'économie, etc. Les membres de ce Comité, tous siégeant au Parlement fédéral et tous fédéralistes, s'y montrent sympathiques à la cause du bilinguisme et vont jusqu'à souscrire “à l'objectif global qui consiste à faire du français la langue de travail au Québec" (p. 22).

Leur recommandation sur la politique sociale, bien qu'intéressante, demeure équivoque, mais tel n'est pas le cas de leur position sur la politique économique: elle aboutit à accorder la prépondérance au gouvernement fédéral et à renforcer encore la centralisation du système. Du point de vue québécois, le plus grave reproche qu'on peut faire à ce rapport - et qu'on lui a fait en réalité - est celui d'avoir négligé de répondre à la question qui pourtant avait donné naissance au Comité: la question du Québec ou comment intégrer le Québec nouveau dans le Canada d'aujourd'hui. Aussi, deux membres, deux Québécois francophones, les députés Martial Asselin et Pierre Debané, se sontils vus obligés de rédiger un rapport minoritaire, dans lequel ils ont accusé le Comité de passer à côté de la question, c'est-àdire de la place à donner au Québec dans la Confédération.

Du point de vue proprement constitutionnel, il s'agit d'un ouvrage très riche, dont les recommandations suivent d'assez près les propositions de la Charte de Victoria (1971). Du point de vue politique, en dépit de quelques concessions intéressantes dans les domaines linguistique et social, il n'offre rien de neuf qui permettrait au Québec de se sentir chez lui et accepté tel qu'il est dans le nouveau régime constitutionnel que propose le Comité.

Ainsi, le monde des écrivains et des intellectuels anglophones s'inquiète beaucoup de l'avenir, mais c'est avant tout et surtout

${ }^{7}$ Le Comité spécial mixte du Sénat et de la Chambre des communes sur la Constitution du Canada. Rapport final (Quatrième session de la vingt-huitième législature, 1972). Edition bilingue, $128 \mathrm{p}$. en français et 128 p. en anglais. 
de l'avenir du Canada; s'il s'intéresse au Québec, c'est parce que cette province menace aujourd'hui de briser l'unité du pays et de faire sans lui son propre avenir.

\section{II - LA CONTRIBUTION FRANCOPHONE}

Du côté francophone, la moisson, sans être aussi abondante, n'en est pas moins intéressante, d'autant plus qu'elle comporte une collaboration étrangère. La demi-douzaine de volumes à signaler peut se partager ainsi: deux ouvrages généraux, un dossier, deux analyses critiques et un plaidoyer.

\section{Les ouvrages généraux}

J'entends par là des ouvrages qui traitent du fédéralisme en général, en formulent la théorie et en examinent les applications concrètes, au nombre desquelles ils rangent le cas du Canada. Tel est, par exemple, l'ouvrage collectif publié à Montréal sous le titre Fédéralisme et nations ${ }^{8}$. Différents auteurs (André Bernard, Jacques Lazure, Claude Corbo) y développent d'abord la théorie du fédéralisme, puis d'autres procèdent à l'étude des cas concrets. En tout, neuf cas: les fédéralismes canadien, suisse, soviétique, yougoslave, tchécoslovaque, malaisien, nigérian, congolais et camerounais. J'avoue que, pour ma part, j'ai été déçu du peu de place que l'ouvrage accorde au fédéralisme canadien. Pas de présentation générale, mais uniquement sous le titre, Le Québec et le fédéralisme, 1950-1970, une "chronique des débats idéologiques et des événements politiques" survenus durant ces vingt ans, le tout se terminant par une analyse détaillée des résultats des élections du 29 avril 1970. L'histoire y trouve son compte, sans doute, mais, quoi qu'en dise le responsable de cet ouvrage collectif, Roman Serbyn, il y aurait eu, me semble-t-il, avantage et intérêt à utiliser pour le cas canadien le même schéma qu'on retrouve dans les autres cas étudiés ${ }^{9}$. A cause du sujet, du titre et des cas traités, un

8 Roman Serbyn, éd., Fédéralisme et nations, coll. "Les Cahiers de l'Université du Québec" (Montréal, Les Presses de l'Université du Québec, 1971), $290 \mathrm{p}$.

9 Voici ce qu'écrit dans l'Avant-propos le responsable du volume: "L'intention principale des collaborateurs de ce Cahier est de fournir au lecteur un ensemble d'études sur la théorie et la pratique des systèmes fédéraux dans les pays multinationaux... Dans l'ensemble des cas, les auteurs examinent les structures et l'évolution de la fédération étudiée. Les grands traits du fédéralisme canadien étant familiers au lecteur canadien en général et au lecteur québécois en particulier, nous avons cru utile de lui présenter une étude qui ne suit pas le schéma habituel" (7-8). 
volume de ce genre mériterait une diffusion internationale, mais les lecteurs des autres pays en apprendront moins sur les structures et l'évolution de la fédération canadienne que sur celles des huit fédérations étrangères dont le volume s'occupe. Dans les articles dits de "théorie", et donc de portée universelle, je me contente de signaler celui qui m’a le plus intéressé: "Le fédéralisme dans les pays multinationaux: avantages et limites", par André Bernard.

Dans l'autre ouvrage de la même catégorie, la place faite au fédéralisme canadien est encore plus restreinte: une dizaine de pages environ ${ }^{10}$. L'auteur est-il canadien, québécois ? Rien ne me permet de l'affirmer. Il a pris pour sujet de thèse l'aménagement constitutionnel des Etats de peuplement composite, Etats qui peuvent être soit unitaires soit fédératifs. Aux problèmes qui assaillent ces Etats de peuplement composite, diverses solutions ont été données: territoriales et personnelles, les premières se retrouvant aussi bien dans le cadre de l'Etat unitaire, comme en Belgique et en Afrique du Sud, que dans les Etats à régime fédératif, comme au Canada et en Suisse.

Ce qui intéresse l'auteur, Bernard Touret, c'est de préciser les rapports entre "la pluralité" et le fédéralisme. Il distingue à ce sujet trois cas: le cas où la pluralité est étrangère à la formation du système fédéral et il donne comme exemple: la Suisse; le cas où la pluralité n'a exercé qu'une influence partielle sur la formation de l'Etat fédéral, et les exemples en sont le Canada et l'Union indienne; le cas, enfin, où l'influence de la pluralité sur la formation du système fédéral a été déterminante, comme en témoignent les exemples soviétique et yougoslave. Examinant ensuite le rôle de la pluralité dans la détermination des unités fédérées, il distingue deux cas: le cas où l'unité fédérée est une entité historique, par exemple, la Suisse qui est une fédération de cantons, et le Canada, qui est une fédération de provinces; le cas où l'unité fédérée est une entité nationale ou linguistique, par exemple, en plus de l'Union indienne actuelle, l'U.R.S.S. et la Yougoslavie, qui sont des fédérations de nationalités.

10 Bernard Touret, L'aménagement constitutionnel des Etats de peuplement composite. Publié pour le "Centre international de Recherches sur le Bilinguisme" (Les Presses de l'Université Laval, Québec, 1973), 260 p. Dans la même série et au même endroit, il faut signaler l'ouvrage très instructif de Jean Falch, Contribution à l'étude du statut des langues en Europe (Québec, 1973), 280 p. 
Bien que plus juridique et politique que vraiment historique et bien que ne traitant du fédéralisme que comme un des moyens de résoudre les problèmes qui surgissent dans les Etats à peuplement composite, cet ouvrage mérite une attention particulière de la part des Canadiens qui aimeraient savoir quelles solutions les autres pays ont données au problème de la cohabitation des divers groupes ethniques, linguistiques et culturels sur leur territoire.

\section{Un dossier}

Avec le petit volume de Jean-Pierre Prévost, directeur du Service d'Information de la Chambre de Commerce internationale, nous abordons franchement le probième du fédéralisme canadien ${ }^{11}$. C'est un dossier, un recueil documentaire, ayant pour but d'initier le lecteur français au fonctionnement du fédéralisme canadien ainsi qu'aux difficultés qu'il rencontre présentement. Dans une introduction d'une quinzaine de pages, l'auteur décrit la situation à la fois historique, sociologique, politique et juridique de notre régime fédératif, description qu'il développe sous quatre titres: une constitution pragmatique, le renforcement des provinces, le défi du Québec et la Charte mort-née de Victoria. "Le fédéralisme canadien, y lit-on, a été inventé en 1867 dans des conditions guère agréables pour les amateurs de systèmes et les théoriciens. Essentiellement pragmatique, il fut davantage l'œuvre des hommes d'affaires que des juristes et répondait en fait à des préoccupations beaucoup plus économiques que politiques (p. 7)."

A la suite de cette Introduction, dans l'ensemble bien faite et objective, commence l'initiation par les textes, textes tirés de l'histoire canadienne, en particulier des déclarations des hommes politiques et groupés en huit chapitres: 1) le régime constitutionnel (les principaux articles de l'Acte de 1867), 2) la "structure de liaison" actuelle, 3 ) les aspects économiques et financiers, 4) le système de partis, 5) le débat sur le fédéralisme, 6) la modification de la Constitution, 7) la Charte de Victoria, 8) le partage en matière sociale. Après une brève présentation de chaque chapitre, l'auteur laisse parler les textes. Il s'agit bien d'un recueil documentaire, dans lequel, par exemple, j'ai été heureux de retrouver au complet le texte de la Charte de Vic-

11 Jean-Pierre Prévost, La crise du fédéralisme canadien, coll. "Dossiers Thémis" (Presses Universitaires de France, Paris, 1972), 96 p. 
toria ainsi que celui qui a exprimé, lors de la même conférence fédérale-provinciale de 1971, la position du Québec en matière sociale.

\section{Les analyses critiques}

Nous passons ensuite à des ouvrages d'histoire, mais d'une histoire bien particulière, écrite par quelqu'un qui a participé aux événements qu'il raconte et qui, fort de son expérience passée, entreprend de jeter un coup d'œil sur l'avenir: Claude Morin, sous-ministre pendant plus de huit ans, de juin 1963 à octobre 1971, et principal conseiller du gouvernement du Québec en matière de relations intergouvernementales ${ }^{12}$. A noter que, dans ces deux volumes, il ne s'agit pas de présenter et d'étudier le fonctionnement du fédéralisme canadien en général, mais uniquement la situation du Québec dans la Confédération, surtout en ces dernières années.

Le premier volume, intitulé Le pouvoir québécois... en négociation, débute par cette question: "Quels gains le Québec a-t-il réalisés depuis dix ans ?'. La réponse nous est donnée 180 pages plus loin et après dix chapitres, constituant autant de dossiers et portant chacun sur un point précis, un problème particulier. Par exemple: le régime de rentes du Québec, les programmes conjoints, le partage fiscal, les relations France-Québec, les conférences et organisations internationales, la révision constitutionnelle, etc. L'auteur avertit, dès le début, que son livre n'est pas seulement un assemblage de dossiers, mais qu'il peut aussi "constituer un instrument de travail et de référence sur une période de l'histoire politique du Québec où la documentation factuelle fait encore terriblement défaut" (p. 8). Et il a raison. Ceux qui s'intéressent à l'histoire de cette période, en particulier aux luttes du Québec pour s'affirmer lui-même face à un gouvernement central toujours enclin à la domination, devront lire ces pages lucides et fort éclairantes. Ils y apprendront, entre autres choses, s'ils ne le savaient pas déjà, que "la plupart des "gains" québécois considérés comme significatifs à l'époque ont été réalisés non pas dans des domaines jusque-là fédéraux, mais dans des secteurs provinciaux qu'avec le temps et à l'aide de son pouvoir de dépenser Ottawa avait fini par occuper ou qu'il s'apprêtait à contrôler..." et que, "depuis une dizaine d'années,

12 Claude Morin, Le pouvoir québécois ... en négociation (Les Editions du Boréal Express, Montréal, 1972), 208 p. - Le combat québécois (ibid., 1973), $192 \mathrm{p}$. 
non seulement les "gains" québécois n'ont pas valu au Québec d'accroissement substantiel de sa force politique, mais les problèmes qui se posent entre Ottawa et Québec demeurent plus nombreux que jamais" (p. 190).

Faisant directement suite au premier et le complétant, le second ouvrage de Claude Morin, Le combat québécois, débute par une série de "bulletins de nouvelles", à la manière du Boréal Express, allant de 1972 à 2002, révélant d'année en année les progrès de l'invasion fédérale et se terminant par l'annonce de l'établissement prochain d'un gouvernement unitaire et par la proclamation de l'anglais comme seule langue officielle du Canada. L'auteur s'efforce ensuite de répondre aux six questions suivantes, lesquelles, précise-t-il, expriment la dynamique du fédéralisme tel qu'il existe: 1) les positions politiques québécoises et fédérales sont-elles conciliables ? 2) dans quelle mesure le Québec peut-il trouver des appuis auprès des autres provinces ? 3) en quoi peut-on compter sur les ministres et députés fédéraux du Québec ? 4) quel appui peut-on espérer de l'opinion publique québécoise ? 5) le Québec peut-il faire confiance au régime fédéral pour assurer l'équilibre entre Ottawa et les provinces ? 6) qu'attendre d'une révision constitutionnelle en profondeur?

Les réponses de l'auteur à ces questions ne sont guère de nature à engendrer l'optimisme pour l'avenir. Selon lui, le conflit Québec-Ottawa ne provient pas seulement du fait qu'on ne s'entend pas sur la répartition des pouvoirs. Il ne s'agit là que d'une manifestation du conflit. La cause en est plus profonde. "Elle réside dans la répugnance fort compréhensible qu'éprouve le Canada anglais à accepter des objectifs québécois dont la réalisation, même incomplète, entraînerait pour lui la transformation à plus ou moins brève échéance d'une structure politique et administrative dont il s'accommode fort bien et qui convient à ses propres aspirations" (p. 175).

L'auteur, on le sait, a opté pour l'indépendance politique du Québec, et cela surtout après son expérience pratique du fédéralisme canadien, laquelle expérience l'a convaincu que "le véritable risque pour la nation québécoise ne se trouve pas dans l'indépendance, mais dans sa soumission à des forces politiques qui la conduisent et la conduiront de plus en plus à se comporter chez elle en minorité culturelle" (p. 182). Les faits, les exemples, les explications et les considérations qui remplissent ces deux ouvrages feront certainement réfléchir tous ceux qui les liront, même ceux qui ne partagent pas cette option. 


\section{Un plaidoyer}

A ces analyses critiques de Claude Morin tente de répondre le plaidoyer de Gilles Lalande ${ }^{13}$. Plaidoyer d'abord en faveur du fédéralisme en général, dont l'auteur, dans une première partie, décrit la popularité, la variété, les principes opératoires et les avantages, points sur lesquels je me sens assez d'accord, ayant déjà plusieurs fois écrit sur le même sujet (voir, par exemple, ma brochure sur Le fédéralisme, publiée en 1951).

Sa deuxième partie, cependant, prête beaucoup plus à controverse. Abordant "la remise en question du fédéralisme au Québec", il entend répondre aux objections les plus courantes qui circulent au Québec contre le régime fédératif canadien. Selon lui, cette remise en question du fédéralisme part d'une méconnaissance profonde du fédéralisme en tant que mode privilégié d'organisation politique de la société moderne. De plus, "les attaques anti-fédéralistes les plus courantes au Québec reposent par ailleurs en bonne partie sur une interprétation historique et sur une connaissance de la réalité sociologique québécoise dont le moins qu'on puisse dire est qu'elles sont éminemment discutables". Enfin, "cette contestation s'appuie largement sur une kyrielle de thèses et d'affirmations globables qui n'ont pour toute justification que celle de s'appuyer sur l'état d'infériorité économique incontestable du groupe canadien-français" (p. 94). Paroles qui rejoignent celles qu'on retrouve dans l'Avant-propos, où l'auteur affirme qu'il entend montrer “comment la majeure partie des propos tenus au Québec à l'égard du régime fédératif canadien repose soit sur des affirmations simplistes, soit sur des conceptions insuffisamment étendues et par trop exclusives du fédéralisme, soit sur des déclarations qui ne sont pas toujours exemptes d'opportunisme et de partisanerie" (p. 12).

Dans une troisième partie, l'auteur s'efforce de montrer que le fédéralisme est la formule "la plus apte à servir les intérêts durables du Québec et des Canadiens français", qu'il constitue "une réponse logique à la condition minoritaire des Canadiens français en Amérique" et qu'il "apparaît comme l'instrument par excellence de l'avenir, en raison de son extraordinaire souplesse". En principe ou en théorie, il a raison; en pratique,

13 Gilles Lalande, Pourquoi le fédéralisme. Contribution d'un Québécois à l'intelligence du fédéralisme canadien, Coll. "Constantes" (Hurtubise/ HMH, Montréal, 1972), $208 \mathrm{p}$. 
demeurent toujours les faits mis de l'avant par Claude Morin dans ses deux volumes et qui visent à démontrer que le régime fédératif a servi les intérêts des Anglo-Canadiens et contribué à réduire les francophones au rang de minorité culturelle même au Québec. C'est dire qu'entre la théorie et la pratique du fédéralisme, il y a loin. Pour ma part, je suis porté à penser que ne font pas le poids les réponses de Gilles Lalande aux critiques formuléees à l'égard du régime fédératif canadien, en particulier par Claude Morin, et qu'en conséquence elles ne réussiront à convaincre que ceux qui sont déjà décidés à ne pas en tenir compte.

Le débat sur le fédéralisme canadien se continue. Les années 1971-1973 l'ont alimenté de nombreux ouvrages, dont quelquesuns de grande valeur. Il se poursuivra vraisemblablement aussi longtemps que "les deux peuples fondateurs" de la Confédération auront à vivre ensemble sous un même régime politique.

RICHARD ARÈs, s.j. 